\title{
A methodology to discover and understand complex patterns: Interpreted Integrative Multiview Clustering ( $\left.\mathrm{I}^{2} \mathrm{MC}\right)$
}

\author{
Beatriz Sevilla-Villanueva*, Karina Gibert, Miquel Sànchez-Marrè \\ Knowledge Engineering and Machine Learning Group (KEMLG), Universistat Politècnica de Catalunya - BarcelonaTech (UPC), Jordi Girona 1-3, Barcelona 08034, Spain
}

\section{A R T I C L E I N F O}

Article history:

Received 29 June 2016

Received in revised form 3 January

2017

Accepted 10 February 2017

Available online $\mathrm{xxx}$

\section{Keywords:}

Multiview Clustering

Crossing clustering

Nutritional studies

Cluster Interpretation

Automatic profiling

\section{$M S C$ :}

41A05

$41 \mathrm{~A} 10$

$65 \mathrm{D} 05$

$65 \mathrm{D} 17$

\section{A B S T R A C T}

The main goal of this work is to develop a methodology for finding nutritional patterns from a variety of individual characteristics which can contribute to better understand the interactions between nutrition and health, provided that the complexity of the phenomenon gives poor performance using classical approaches. An innovative methodology based on a combination of advanced clustering techniques and consistent conceptual interpretation of clusters is proposed to find more understandable patterns or clusters. The Interpreted Integrative Multiview Clustering $\left(\mathrm{I}^{2} \mathrm{MC}\right)$ combines the previously proposed Integrative Multiview Clustering (IMC) with a new interpretation methodology NCIMS. IMC uses crossing operations over the several partitions obtained with the different views. Comparison with other classical clustering techniques is provided to assess the performance of this approach. IMC helps to reduce the high dimensionality of the data based on multiview division of variables.

Two innovative Cluster Interpretation methodologies are proposed to support the understanding of the clusters. These are automatic methods to detect the significant variables that describe the clusters; also, a mechanism to deal with the consistency between the interpretations inter clusters of a single partition CI-IMS, or between pairs of nested partitions NCIMS. Some formal concepts are specifically introduced to be used in the NCIMS.

$\mathrm{I}^{2} \mathrm{MC}$ is used to validate the interpretability of the participant's profiles from an intervention nutritional study. The method has advantages to deal with complex datasets including heterogeneous variables corresponding to different topics and is able to provide meaningful partitions.

(C) 2016 Published by Elsevier Ltd.

\section{Introduction}

The paradigm of Health Science is evolving towards personalized medicine, and nutrition habits are crucial for healthy life styles promotion. Traditionally, dietary intervention studies have been conducted to understand why similar diets have different effect on apparently similar people. These studies aim to find the effect of nutrients over the participants by comparing the state of a person before and after the interventions. These are highly complex studies, since they mix a large number of variables for describing the participants. These variables have different origins such as anthropometric and socio-demographic data, habits recording, and clinical blood, urine and genetic tests. In addition, the interactions between the diet or other environmental factors and genes are very complex and difficult to detect by classical modeling methods. There are additional sources of complexity: among them, the relatively small effect of dietary interventions on physiological parameters; nutrition effects on gene-expression patterns are also hard to detect ([2]); also, as the intervention ef-

\footnotetext{
* Corresponding author.

Email address: bea.sevilla@gmail.com (B. Sevilla-Villanueva)
}

fect has not specific target, it can be reflected in multiple bio-markers.

The classical pre-post intervention studies approach considers numerical variables, compute differences and search for a model relating the diet with those differences. Until now, these techniques have not been expressive enough to allow the extraction of complex relationships in nutritional intervention studies, given that the level and degree of interactions between different subsets of variables is too complex, within this context, to be captured by simple data analysis or pre-post statistical testing.

In this paper, it is proposed to previously identify participants' profiles suitable for finding intervention effects locally to each type of individual.

Clustering of participants is difficult since the big amount of variables include a wide range of aspects and not all variables play the same role. Thus, the Interpreted Integrative Multiview Clustering $\left(\mathrm{I}^{2} \mathrm{MC}\right)$ methodology is proposed to get a profiling of participants properly conceptualized.

The transfer of knowledge is crucial in interdisciplinary contexts. Hence, the interpretation of the resulting clusters is essential for further decisions. Cluster Interpretation is the post-process of finding the common and distinctive characteristics of every cluster and expressing them in a conceptual synthesis, directly understandable. However, this is still an open issue from the methodological point of view ([37]). In previous works, authors developed the use of statisti- 
cal tests to select the significant variables in the clusters ([38]) by introducing the Test-Value in the interpretation process (more sensible and informative than other classical tests). Nevertheless, tests are sensible to the cluster size and produce some interpretative inconsistencies. In [21] a modification of the Test-Value is introduced to avoid inconsistent interpretations due to the size differences among clusters. Also, the consistency between interpretation of nested partitions is analyzed.

In the present work, these partial results are refined and integrated in two formal interpretation new methodologies. To do that, new elements have been introduced in this work: 1) a formalization of the concept of cluster descriptor providing the language over which interpretations will be built; 2) a generalization of Test-Value to variable sample sizes to evaluate robustness of results; 3 ) a formal and general Sensitivity Analysis method that uses the generalized Test-Value to assess robustness of cluster descriptor to variations of sample sizes; 4) the formalization of the concept of description-power of a cluster descriptor that graduates its robustness according to the Sensitivity Analysis results; 5) the introduction of a special type of basic descriptors for quasi-constant variables inside a cluster that cannot be assessed by significance tests; 6) a regular-expression-based method to transform formal cluster descriptors into conceptual description of a cluster.

The two new interpretation methodologies proposed are: CI-IMS (Cluster Interpretation based on Integrated Marginal Significance), providing automatic conceptual description of a given partition by guaranteeing both consistency of concepts inter-clusters and stability of results. NCI-IMS (Cluster Interpretation based on Integrated Marginal Significance for nested partitions), providing automatic conceptual description of a given pair of nested partitions by guaranteeing consistency inter-parts, consistency inter-partitions and stability of results. NCI-IMS uses CI-IMS inside and combines CI-IMS interpretations of both nested partitions.

CI-IMS and NCI-IMS integrate the Test-Value and the proposed sensitivity analysis to find cluster descriptors with a certain description-power for giving conceptual interpretations.

On top of that, the $\mathrm{I}^{2} \mathrm{MC}$ combines IMC ([39]) to build the profiles, which intrinsically produce a final partition nested in some intermediate ones created by the IMC itself, and gives consistent and robust interpretation for final and intermediate partitions by using NCI-IMS.

In this work, data from a trial study of the Mediterranean diet effect is used to show the benefits of the presented proposal.

The structure of this paper is: background on Multiview Clustering approach, clustering in real nutritional studies and Cluster Interpretation is presented in Section 2; the proposed $\mathrm{I}^{2} \mathrm{MC}$ methodology is detailed in Section 3 together with specific details on the different steps, by including all the new concepts introduced to formalize the two Cluster Interpretation methods proposed CI-IMS and NCI-IMS ; then, the case study and the results are presented; finally, conclusions and future work.

\section{Background}

Clustering tries to find hidden structure in unlabeled data. The aim of clustering is to group a set of objects into distinct clusters of similar objects. The similarity used varies from one method to another ([12]). Currently, there are multiple clustering approaches most with some variants.

The Multiview Clustering deals with the high dimensionality of data. In this approach, the variables are split in several groups. Then, the subjects are clustered under each group of variables or view. In [6], variables are split in two independent groups by their meaning. In
[43], the user specify the views through some keywords such that a query system retrieves all relevant variables to that keyword in the database. In cross-clustering, separated views are build as multiple Hierarchical Bayesian Clustering using a single variable each; the several views are merged uncer certain criteria in a final single clustering [32].

The need of finding consensus techniques to combine the various clusterings obtained under Multiview Clustering is stressed by [1]. Ensemble methods and semi-supervised techniques are proposed among others. Authors claim that most of the effort must concentrate on the agreement between the different views or resulting partitions.

As said before, clinical studies use traditional basic hypothesis testing or statistical modeling. Clustering is not very popular in this area. Few works are found in the field using clustering. In [27], K-means is used with dietary variables and other lifestyle factors using K-means. In [41], a two-step cluster analysis based on diet and physical activity revealed two distinct clusters: obesogenic and non-obesogenic. Even though few works are found, the use of clustering for finding patterns of response to diet may be of help to assist decision making in diet design and finding the best target population for a specific type of food or dietary pattern.

In many data mining processes there is still a gap between raw data mining results and effective decision-making. Some works point to this issue $[10,19]$ and, for the particular case of clustering, interpretation and conceptualization of clusters is key to this purpose. Some previous works give some ideas $[3,15-17,40]$. Cluster Interpretation is usually made by examining the cluster centroids (averages inside each cluster). Clusters are distinguishable only if some variables show means significantly different in some clusters [36], often assessed with independent $t$-test or ANOVA. In [8], the variables used in the clustering are ranked with the $\log$ Worth index based on $\chi^{2}$-Independence Test.

\section{Methodology}

In this work, Interpreted Integrative Multiview Clustering ( $\left.{ }^{2} \mathrm{MC}\right)$ is proposed to find conceptual patterns on a set of individuals. The variables collected in the data set can describe different aspects of the persons like intrinsic characteristics or physical activity. The idea is to split the original set of variables into different subsets according to the semantics of the variables. These subsets can be analyzed locally, as in original Multiview Clustering approach, this addressing the high dimensionality. The variable's space is split in different subsets that play different roles in the analysis, named here thematic blocks. Domain knowledge is used to identify the different roles of the variables and to define the thematic blocks. These blocks are made in such a way that each one regards a different aspect of the individuals. Then, individuals are clustered using a single thematic block each time, generating independent views.

However, giving several disconnected views of the data, as classical Multivew proposes, do not help to decision-making. Thus, IMC ([39]) extends the Multiview Clustering by an integrative step where the results of each view are properly combined to give a single partition combining the structure found under all views (see Fig. 1).

The result of IMC is a set of partitions corresponding to cluster each thematic block and a final partition integrating all the thematic partitions. The final partition is nested by construction to the thematic ones. To understand the meaning of the resulting clusters, the NCI-IMS is used for interpretation that guarantees the consistency between the interpretation of all partitions.

The proposed I IMC is as follows. Given:

$-\mathscr{I}=\left\{i_{1}, \ldots, i_{n}\right\}$ a set of individuals

- $\mathscr{X}$ a set of variables describing the individuals of $\mathscr{I}$ 


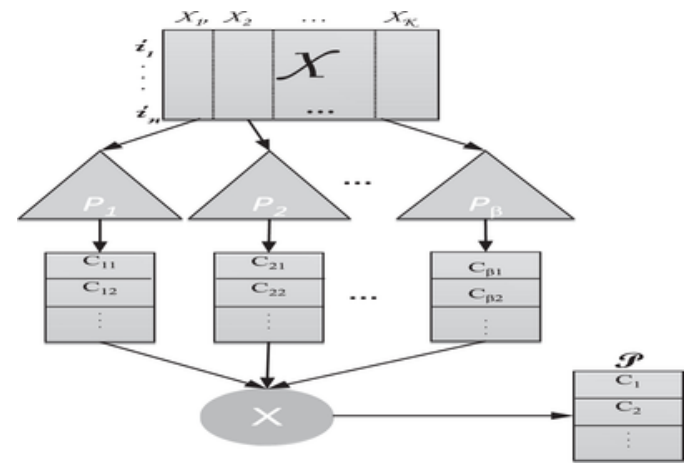

Fig. 1. Integrative Multiview Clustering Scheme.

1. Data Preprocessing: real data require a preprocessing step to prepare them for the analysis. The dataset is treated with common methods like variable's selection, correction of data errors and inconsistencies whenever possible, creation of composite indicators, aggregations, recodifications, missing data treatment ([20]). The partition resulting from clustering do not contain missing values and need not specific treatment before interpretation step.

2. Determine the Thematic Blocks:

Divide $\mathscr{X}$ variables in a small number of thematic blocks using the background expertise in the area. Ideally one thematic block per topic referred in the data set is recommended. $\mathscr{B}=\left\{\mathscr{B}^{1}, \mathscr{B}^{2}, \ldots, \mathscr{B}^{\beta}\right\}$ such that: $\forall b \in \mathscr{B}: b \subseteq \mathscr{X}$ it holds that: i) $\bigcup_{\forall b \in \mathscr{B}} b \subseteq \mathscr{X}$; ii) $\forall_{b, b^{\prime} \in \mathscr{B}} b \cap b^{\prime}=\emptyset$

3. Profiling of the individuals:

3.1 Integrative Multiview Clustering over each block $\mathscr{B}^{1}, \mathscr{B}^{2}, \cdots, \mathscr{B}^{\beta}$ getting an integrated partition $\mathscr{P}$ :

i. For each block from $b \in \mathscr{B}$, cluster using the variables in the block (see section Section 3.1) and get a partition of $\mathscr{I}$ namely $\mathscr{P}_{b}$. This produces several partitions of $\mathscr{I}: \mathscr{P}_{1}, \mathscr{P}_{2}, \cdots, \mathscr{P}_{\beta}$.

ii. Build the integrated partition $\mathscr{P}=\mathscr{P}_{1} \times \mathscr{P}_{2} \times \cdots \mathscr{P}_{\beta}$.

Formally, there is no limitation on the number of thematic blocks built in step 2 . However, from a practical point of view $\beta>5$ can produce very sparse cartesian products at this step and it is recommended to keep $\beta$ smaller.

3.2 Conceptualization of clusters: characterize and interpret the clusters of $\mathscr{P}$ by combining the interpretations of $\mathscr{P}_{1}, \mathscr{P}_{2}, \cdots, \mathscr{P}_{\beta}$ under the NCI-IMS interpretation methodology (Section 3.11). This helps to understand the concept behind the cluster. Then, experts can assign an identifier label to each cluster, which catches the conceptualization done.

In this paper, the result of $\mathrm{I}^{2} \mathrm{IMC}$ is evaluated and validated:

1. Evaluate the goodness of the partition $\mathscr{P}$ : use the Dunn-like cluster-validity index (see Section 3.4).

2. Validation: validate the performance of the IMC by comparing the resulting partition $\mathscr{P}$ with other partitions obtained by other clustering methods.

Cluster $\mathscr{I}$ using the variables in $\mathscr{X}$ by means of: Hierarchical Clustering with Ward's method and partitional method PAM.

Compare with $\mathscr{P}$ at two levels:

- Structural: evaluate all the resulting partitions using the Dunn-like cluster-validity index.

- Interpretability: compare the interpretations provided by the NCI-IMS methodology between $\mathscr{P}$ and the one getting the best Dunn-like index.

\subsection{Hierarchical Clustering}

Advantages of Hierarchical Clustering include ([4]): embedded flexibility regarding the level of granularity, easy handling of any distance, applicability to any variable types and no need to predefine the number of required clusters.

An agglomerative Hierarchical Clustering with Ward's method ([42]) is used. It finds compact and spherical clusters ([9]). It merges clusters by minimizing inter-clusters variance loss. It is widely used since it is related to the quantity of information of the clusters, and results often are easier to interpret than those obtained with other methods.

The number of clusters is determined by cutting the dendrogram at the horizontal level maximized by the Calisnki-Harabasz Index ([7]).

\subsection{Gower's dissimilarity coefficient}

All clustering methods are distance-based methods, where comparisons between objects are used to guide clusters formation. Most of the works referred before on clustering with nutritional data refers to numerical data. However, as soon as clinical information is taken into account, qualitative variables may be included in the analysis. Since data are heterogeneous, a mixed metrics is used. There are many possibilities in the literature, such as [24], [23], [18], [28], [34], [35] and [22].

Gower's coefficient used in this work considers numerical, qualitative, ordered discrete or binary variables ([11]). Briefly, Gower's dissimilarity combines a min-max normalized distance for numerical variables with equality for qualitative variables, by taking into account missing data. [24] demonstrates that the square of the original dissimilarity is a metrics, being suitable for Hierarchical Clustering.

\section{3. $P A M$}

PAM is a partitional clustering algorithm ([29]) that minimizes the overall distance between cluster representative and its members. It is more robust than $\mathrm{k}$-means since it uses medoids instead of centroids and the underlying distance is not restricted to be $L_{2}$-norm.

\subsection{The Dunn-like index}

The Dunn-like index $(D)$ is a generalization of Dunn index ([13]). It measures the trade-off between the cohesion of the clusters and their separation. It attempts to identify compact and well-separated clusters. From all generalizations of the Dunn index $([5,33])$, the one available in FPC-R package of [26] substitutes point to point distances index by average inter-clusters distance in numerator, and average intra-clusters distance in the denominator, thus being more robust ([25]).

$$
D=\frac{\min _{C, C^{\prime} \in P} \overline{\delta_{c, c^{\prime}}}}{\max _{C \in P} \overline{\Delta_{c}}} ; \quad \overline{\delta_{c, c^{\prime}}}=\frac{\sum_{i \in C, i^{\prime} \in C^{\prime}} d\left(i, i^{\prime}\right)}{n_{c^{n} c^{\prime}}} ; \quad \overline{\Delta_{c}}=\frac{\sum_{i, i^{\prime} \in C} d\left(i, i^{\prime}\right)}{n_{c}\left(n_{c}-1\right)}
$$

\subsection{Generalized Test-Value}

The original Test-Value was introduced by [31] to identify the significant variables (descriptors) in the several clusters of a partition, as 
a support to understand the clusters's meaning. This test is based on global versus cluster's means difference and it is sensible to the cluster size ([21]). Thus, the same means difference is significant or not depending on the cluster size and can generate inconsistent interpretations of different clusters. A generalization is needed for a consistent interpretations among clusters of different sizes.

Hence, the Test-Value is parameterized to evaluate all clusters under the same reference: the cluster size from the original definition of Test-Value, is substituted by a parameter $v$. The new equations of Generalized Test-Value for numeric variables and for categories of qualitative variables are in Table 1 . Thus, $\tau_{v}$ and $\pi_{v}$ assesses significant differences between the clusters and the global population assuming a common cluster size $v$ that in [21] is proposed to be the total sample size $(v=n)$. From now, $\Theta=\left\{\tau_{v}, \pi_{v}\right\}$ refers any of these two tests.

\subsection{Basic descriptors}

A specific treatment is deserved for variables that are constant in a cluster, as they have null variance and tests cannot be assessed. In fact, these variables are intrinsic descriptors of the cluster by themselves. For that reason, the concept of basic descriptors is introduced here. Basic descriptors are a relaxation of variables constant in a cluster, admitting a certain level of variability (namely $\delta$ ). Given a qualitative variable $X$, a category $s \in \mathscr{D}_{X}$ and a cluster $C$, $s$ is a basic descriptor of $C$ iff: $\left(p_{s c} \geq 1-\delta\right) \vee\left(p_{s c} \leq \delta\right)$, being $p_{s c}=P(s \mid C)$. Dominant categories of any qualitative variable (with probability greater than $1-\delta$ in the cluster) are considered. The absence of a category is also considered (probability lower than $\delta$ ).

\subsection{Sensitivity Analysis}

Given a partition $P$, the proposal is to evaluate the Generalized Test-Value with a common $v$ for all clusters in $P$. In ([21]) it was proposed to use $v=n$. Since the Generalized Test-Value depends on $v$, the significance of a certain variable clearly depends on cluster size, which can vary.

As a consequence, variations in $v$ can change the results of the tests, according to the intrinsic performance of significance tests ([21]). Robustness of significance to small or large variations of $v$ must be assessed to evaluate the pertinence of relying in the $p$-values to build interpretations. For this purpose, a Sensitivity Analysis is introduced to graduate a variable according to the stability of their significance to variations of the $v$. Indeed, large differences between local and global means will keep significant even with drastic reductions in sample size and those variables will play a role to interpret the cluster. On the contrary, small differences might present unstable results providing significance or not, in different clusters depending on the sample size, and maybe it will be better to ignore those variables for interpretation.

In this paper a Sensitivity Analysis over 5 different sample sizes is presented, although it can be easily extended to more sample sizes as well. The proposed Sensitivity Analysis (SA method) uses two parameters $\varepsilon_{1}, \varepsilon_{2}$ to built 5 values of $v$ and evaluate robustness of significance at 5 hypothetic sample sizes. Given $\varepsilon_{1}, \varepsilon_{2} \in(0,0.5] \wedge \varepsilon_{1}+\varepsilon_{2}<1$ a sequence of 5 sample sizes is generated: $\mathcal{S}=\left\{v_{1}\right.$

$=n\left(1-\varepsilon_{1}-\varepsilon_{2}\right), v_{2}$

$=n\left(1-\varepsilon_{1}\right), v_{3}$

$=n, v_{4}$

$=n\left(1+\varepsilon_{1}\right), v_{5}$

$\left.=n\left(1+\varepsilon_{1}+\varepsilon_{2}\right)\right\}$

Given a variable $X$; a cluster $C$ and a test $\Theta_{v}$, the idea is to assess $\Theta_{v}$ $\forall v \in \mathcal{S}$. Then, the concept of description-power (dp) of $X$ in $C$ is introduced here as a measure of the reliability of the variable to be used for further interpretation. Description-power is defined in Table $2(\checkmark$ indicates test was significant and $\times$ indicates test was no-significant).

This proposal is valid with any statistical test, provided that the sample size is properly parameterized. In the context of this paper, it will be applied over the Generalized Test-Value.

This proposal is an extension of [21] where a non-symmetric operator defined in the specific context of nested partitions was presented. In this work, the description-power is formalized as a general operator for any kind of variable and partition. Also, the proposal has been extended with the introduction of the new category of basic descriptors.

\subsection{Formalization of a cluster descriptor}

In this section a formalization of the concept cluster descriptor is introduced such that allows homogeneous treatment of numerical and qualitative variables in automatic conceptual interpretations of a partition. Cluster descriptors are expressed in terms of a vocabulary $\mathscr{D}_{\chi}$ composed of words W:

$$
W=\left\{\begin{array}{l}
"{ }^{\prime \prime} \quad X \text { is a numerical variable of } \chi \\
\langle s, X\rangle \quad s \in \mathscr{D}_{X}, X \text { is a qualitative variable of } \chi
\end{array}\right.
$$

There is one $W$ per each numeric variable in $\chi$ (the name of the variable itself) and one compound $W$ per each of the values of a qualitative variable in $\chi$ (the pair of variable and category names). Compound words are required for qualitative variables because $s$ by themselves might be not unique.

Then, the descriptor of a $W$ in a cluster $C$ is defined as

$$
\mathscr{V}_{W C}=\langle W, C, d p, \text { sense }\rangle
$$

where,

- $d p$ is the description-power of $W$ in cluster $C, d p \in \Pi$.

- sense indicates if values of $W$ in cluster $C$ behave significantly over or under the global values, according to test $\Theta$ (Table 1), when suitable. The values of sense are sense $=\{T, \perp, \uparrow, \downarrow\}$; where $\uparrow$ and $\downarrow$ are used to express the sense of the significance for all variables, except for basic descriptors, where the tests cannot be assessed and $\mathrm{T}$ and $\perp$ symbols are used instead. Symbols $\uparrow$ or $T$ mean the variable is higher in the cluster than in the global sample; $\downarrow$ and $\perp$ mean the variable is lower in the cluster than in the global sample. In Section 3.9 details on how to compute it are provided.

Table 1

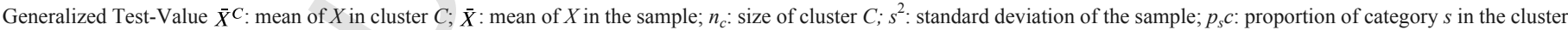

\begin{tabular}{|c|c|c|c|}
\hline$\Theta$ & X Numeric & X Qualitative & \\
\hline & $\tau_{v}=\frac{\bar{X}^{c}-\bar{X}}{\sqrt{\left(1-\frac{n_{c}}{n}\right) \frac{s^{2}}{v}}} \sim t_{v-1}$ & $\pi_{v}=\frac{p_{s c}-p_{s}}{\sqrt{\left(1-\frac{n_{c}}{n}\right) \frac{p_{s}\left(1-p_{s}\right)}{v}}} \sim z$ & (3) \\
\hline
\end{tabular}
$C ; p_{s}$ : proportion of category $s$ in the sample. 
Table 2

Sensitivity Analysis.

\begin{tabular}{llllllll}
\hline \multicolumn{1}{c}{ Sample size } \\
\hline Description ower $\Pi$ & Symbol & $v_{1}$ & $v_{2}$ & $v_{3}$ & $v_{4}$ & $v_{5}$ \\
\hline Robust Non-descriptor & $\bar{R}$ & $\times$ & $\times$ & $\times$ & $\times$ & $\times$ \\
Moderate Non-descriptor & $\bar{M}$ & $\times$ & $\times$ & $\times$ & $\times$ & $\checkmark$ \\
Weak Non-descriptor & $\bar{W}$ & $\times$ & $\times$ & $\times$ & $\checkmark$ & $\checkmark$ \\
Weak escriptor & $W$ & $\times$ & $\times$ & $\checkmark$ & $\checkmark$ & $\checkmark$ \\
Moderate escriptor & $M$ & $\times$ & $\checkmark$ & $\checkmark$ & $\checkmark$ & $\checkmark$ \\
Robust escriptor & $R$ & $\checkmark$ & $\checkmark$ & $\checkmark$ & $\checkmark$ & $\checkmark$ \\
$\checkmark: p-$ value $<\alpha$ & & test $\in \Theta$ & & & \\
\hline
\end{tabular}

\subsection{The CI-IMS methodology (Cluster Interpretation based on Integrated Marginal Significance)}

The CI-IMS methodology combines some of the concepts introduced in previous sections into a general process to automatically build conceptual interpretation of a partition where the conceptualization of all clusters is consistent inter-clusters.

Given a set of variables $\mathscr{X}$ and a partition $P$ :

1. Determine the threshold $\delta \in[0,1]$ to consider a category of a qualitative variable a basic descriptor of a cluster. In principle, a single global $\delta$ is considered for all clusters in a partition for homogeneous treatment of accepted uncertainty. The smaller it is, the better.

2. Determine $\alpha \in[0,1]$ as the significance level of statistical tests involved in the process.

3. Determine $\varepsilon_{1}, \varepsilon_{2} \in(0,0.5] \wedge \varepsilon_{1}+\varepsilon_{2}<1$ for building $\delta$ the set of sample sizes to asses the Sensitivity Analysis.

4. Determine a minimum level of robustness $r \in \Pi$ to accept a cluster descriptor.

5. For all characteristics $\left(\forall W \in \mathscr{D}_{\chi}\right)$ and all clusters $(\forall C \in P)$ :

Build the cluster descriptor $\mathscr{V}_{W, C}=\langle W, C, d p$, sense $\rangle$ as:

(a) for compound $\mathrm{W}$ : if $\mathrm{W}$ is a Basic descriptor (B):

- $p_{s c} \geq(1-\delta) \Rightarrow$ Build $d_{W, C, v}=(\langle s, X\rangle, C, \mathrm{~T})$

- $p_{s c} \leq \delta \Rightarrow$ Build $d_{W, C, v}=(\langle s, X\rangle, C, \perp)$

(b) for simple $\mathrm{W}$ or compound $\mathrm{W}$ non basic descriptor

(1) Find the description-power of $W$ in $C$ by performing SA method $\forall v \in \mathcal{S}$ using the corresponding test according Table 1(test $\tau_{v}$ for simple $\mathrm{W}$ and $\pi_{v}$ for compound $\mathrm{W}$ )

(2) Determine the sense of the cluster descriptor according Table 3.

6. The interpretation of $P$ is $Y_{P}=\left\{\mathscr{V}_{W, C}: \forall W \in \mathscr{D}_{\chi}, \forall C \in P\right\}$.

7. The interpretation of $P$ at level $r \in \Pi$ is $\mathrm{Y}_{P, r} \subseteq \mathrm{Y}_{P}$, as the subset of $\mathscr{V}_{W, C}$ such that all descriptors have at least the level of robustness $r$ :

$$
Y_{P, r}=\left\{\mathscr{V}_{W, C}: d p_{W, C} \geq r\right\}
$$

8. Build the conceptual interpretation of $P$ using a regular expression to translate the cluster descriptors in $\mathrm{Y}_{P, r}$ to a verbose sentence: $\forall \mathscr{V}_{W, C}=\langle W, C, d p$, sense $\rangle \in Y_{P, r}: d p \neq \bar{R}$.

- if $W$ is simple, the regular expression is:

$[X]$ is $[$ sense-name $]$ in the cluster $[C]$

- if $W$ is compound, the regular expression is:

The cluster $[C]$ has [sense

- name $]$ proportion of $[s]([X])$ ' where sense-name takes two possible words in the regular expression: sense - name $= \begin{cases}\text { "higher" " } & \text { if sense } \in\{T, \uparrow\} \\ \text { "lower" " } & \text { if sense } \in\{\perp, \downarrow\}\end{cases}$

9. Visualize the Class Panel Graphs ([17]) using only the subset of variables that appear in $\mathrm{Y}_{P, r}$.

\subsection{Nested partitions and inconsistencies}

A refinement or abstraction of an existing partition gives nested partitions. A refined partition $P_{\ell^{\prime}}$ is nested into a more abstract partition $P_{\ell}$ when $P_{\ell}$ is formed of classes that subdivide the classes of $P_{\ell}$. Let us call superclasses the elements $C$ of $P_{\ell}$ and subclasses the elements $C^{\prime}$ of $P_{\ell^{\prime}}$. Let $S_{C} \subset P_{\ell^{\prime}}$ be the set of subclasses of a class $C \in$ $P_{\ell}$. It holds that $S_{C}=\left\{C^{\prime}: C^{\prime} \in P_{\ell^{\prime}} \wedge C=\left(\bigcup C^{\prime}\right)\right\}$.

IMC, and consequently $\mathrm{I}^{2} \mathrm{MC}$, automatically produces pairs of nested partitions as it combines $\beta$ previous partitions of higher granularity (obtained by independent clustering of the several views) in a Cartesian product that constitutes the final partition provided by the method. The final partition is nested by construction to any of the partitions given by the local views.

In Cluster Interpretation, consistency requires that significant variables in a superclass keep significant in some of its subclasses, as it makes no sense that significant characteristics of a superclass distinguishing the whole superclass from the others, cannot distinguish any of its subclasses from other subclasses.

Then, given a characteristic $W$, a class $C$ and its set of subclasses $S_{C}$, the following situations could occur when comparing the interpretations of $C$ and $S_{C}$ :

1. Specification: subdividing $C$ produces additional information (W) in some subclass $C^{\prime}: W$ is not significant in $C$ and becomes significant in some subclasses $\left(\exists C^{\prime} \in S_{C}\right.$ : Wsignificant in $\left.C^{\prime}\right)$.

2. Irrelevant: subdividing $C$ is not providing additional information: $W$ is not significant in $C$ and keeps non-significant in all subclasses $\left(\forall C^{\prime} \in S_{C}: W \neg\right.$ significant in $\left.C^{\prime}\right)$.

3. Inheritance: the characteristic $W$ has been propagated to some subclass: $W$ is significant in $C$ and keeps significant at least in some subclass $\left(\exists C^{\prime} \in S_{C}\right.$ : Wsignificant in $\left.C^{\prime}\right)$. If $W$ is significant for all subclasses, subdividing $C$ does not provide additional information.

4. Inconsistency: a property that distinguishes the whole $C$ from the global population is not characteristic for any of its subclasses. This is not reasonable and represents an inconsistency: $W$ is significant in $C$, but not significant in any subclass $\left(\forall C^{\prime} \in S_{C}: W \neg\right.$ significant in $C^{\prime}$ ).

With these 4 possible diagnostic situations, the suitability of using $W$ in the description of the superclass and/or in the subclasses can be assessed. Analyzing what happens with all $C^{\prime} \in S_{C}, W$ does not appear in the description of any of the $S_{C}$ subclasses when all subclasses behave as homogeneously non significant; whereas it can appear in some of them or not under a non homogeneous behavior of the subclasses, leading to a specification or inheritance scenario depending on the significance of $W$ in the superclass. Thus, the aggregation is $\forall C^{\prime} \in S_{C}$ in irrelevant and inconsistency situations and it is $\exists C^{\prime} \in S_{C}$ in specification and inheritance situations. This shows that, for specification and inconsistency linked to a $\forall$ operator, the real diagnosis of $W$ cannot be done unless all subclasses are analyzed together, which leads to the introduction of a provisional diagnosis for a single $C^{\prime}$ (see D Table 4). Table 4 defines the aggregation operator for $\mathrm{dp}(\mathrm{D})$. 
Table 4

Provisional diagnosis of descriptor $W$ in nested classes.

\begin{tabular}{lll}
\hline $\mathrm{D}$ & Descriptor superClass & \\
\hline Descriptor ubClass & Non-significant & Significant \\
\hline $\begin{array}{l}\text { Non-significant } \\
\text { Significant }\end{array}$ & $\begin{array}{l}\text { Apparently irrelevant } \\
\text { Specification }\end{array}$ & $\begin{array}{l}\text { Apparently inconsistence } \\
\text { Inheritance }\end{array}$ \\
\hline
\end{tabular}

\subsection{NCI-IMS (Cluster Interpretation based on Integrated Marginal Significance for nested partitions)}

An extension of the interpretation methodology CI-IMS is also proposed to treat the possible inconsistencies among interpretations of nested partitions. The proposed methodology is non-dependent of whether the partitions are known at the same time or a refinement or abstraction is defined later.

Table 4 is extended to the Relations Table ( $\mathscr{R})$ (Table 5). Using the CI-IMS interpretations of both partitions $P_{\ell}, P_{\ell^{\prime}}$, each descriptor of $W$ in a cluster $C \in P_{\ell}$ is linked with a term $\mathscr{V}_{W, C}$, and has an associated description-power $d p_{C}$. The same happens with subclasses in $S_{C}$ (getting $d p_{C^{\prime}} \forall C^{\prime} \in S_{C}$ ). The diagnosis of $W$ regarding $C$ and $C^{\prime}$ must be done considering $d p_{C}$ and $d p_{C^{\prime}}$, rather than significance of $W$ in $C$ and $C^{\prime}$. These determine a cell on $\mathscr{R}$ (Table 5) and provides a provisional diagnosis of $W$ for $C$ and $C^{\prime}$. Thus, Table $\mathscr{R}$ (Table 5) is modeling the potential use of $W$ for describing $C$ or $C^{\prime} \in S_{C}$. This relation table is not symmetric neither commutative.

Now, there is not anymore a binary scenario and the Sensitivity Analysis might produce several evaluations for the subclasses of $S_{C}$. According to that, the role of $W$ as a descriptor seems to change from one cluster to another. This rises the need to introduce an aggregation operator to diagnose the global situation of $W$ regarding the whole $S_{C}$ subset. The aggregation is performed by taking the supreme of all $d p_{C^{\prime}}$, ie the dp of the cluster $C^{\prime} \in \mathcal{S}_{\mathscr{C}}$ where $W$ has more significance.

The problematic situations for consistency between interpretations of $P_{\ell}$ and $P_{\ell^{\prime}}$ come from the cells labeled as inconsistency, even moderate, weak orm limit, as in these situations $W$ seems to be relevant for describing $C$ but disappears from all $C^{\prime} \in S_{C}$ in some sense. One of the contributions of CI-IMS methodology has been to generalize the Test-Value to reduce inconsistencies. Those that persist will be managed at this stage.

Table Actions $(\mathscr{A})$ (Table 6) associates a description decision to be implemented for each situation described in table $\mathscr{R}$. Therefore, a characteristic $W$ will appear or not in the interpretation of the superclass $C$ and in some subclasses of $S_{C}$ or not, according to table $\mathscr{A}$ . Only inconsistencies over certain robustness degree are reported in this description. The resulting 4 possible actions identified are:
- SubC: specification, $W$ will not appear in description of $C$ and will appear in the description of the subclass $C^{\prime} \in S_{C}$.

- None: irrelevance, $W$ will not appear neither in description of $C$ nor $C^{\prime} \in S_{C}$.

- Both: inheritance, $W$ will appear both in description of $C$ and subclass $C^{\prime} \in S_{C}$.

- SupC: inconsistence, $W$ will appear in $C$ but not in the description of the subclass $C^{\prime} \in S_{C}$.

Therefore, the main goal of the NCI-IMS methodology is to produce consistent interpretations of $P_{\ell}\left(Y_{P_{\ell}}\right)$ and the interpretation of a nested partition $P_{\ell^{\prime}}\left(Y_{P_{\ell^{\prime}}}\right)$ in such a way that all relevant characteristics of $P_{\ell}$ appear as relevant characteristics at least in some subclasses in $P_{\ell^{\prime}}$ :

Given $\mathscr{X}$ a set of variables; $P_{\ell}, P_{\ell^{\prime}}$ two partitions such that $P_{\ell^{\prime}}$ is nested in $P_{\ell}$; Table 5 Relations $(\mathscr{R})$; Table 6 Actions $(\mathscr{A})$.

1. Obtain $Y_{P_{\ell}, r}$ the interpretation of $P_{\ell}$ using CI-IMS $\left(\delta, \alpha, \epsilon_{1}, \epsilon_{2}, r, \mathscr{X}\right.$ ) with $r=\bar{R}$

2. Obtain $Y_{P_{\ell^{\prime}}, r}$ the interpretation of $P_{\ell^{\prime}}$ using CI-IMS( $\left.\delta, \alpha, \epsilon_{1}, \epsilon_{2}, r, \mathscr{X}\right)$ with $r=\bar{R}$.

3. $\forall W \in \mathscr{D}_{\chi}, \forall \mathscr{V}_{W, C} \in Y_{P_{\ell}}$ : $\forall \mathscr{V}_{W, C^{\prime}} \in Y_{P_{\ell^{\prime}}}: C^{\prime} \in S_{C}$

$$
\text { - } Y_{P_{\ell}}^{\bullet}=\left\{\mathscr{V}_{W, C} \text { iff } \mathscr{A}\left(d p_{C}, d p_{C^{\prime}}\right) \in\{\text { SupC, Both }\}\right\}
$$$$
\text { - } Y_{P_{\ell^{\prime}}}^{\bullet}=\left\{\mathscr{V}_{W, C^{\prime}} \text { iff } \mathscr{A}\left(d p_{C}, d p_{C^{\prime}}\right) \in\{\text { SubC,Both }\}\right\}
$$

4. $Y_{P_{\ell}}^{\bullet}$ and $Y_{P_{\ell^{\prime}}}^{\bullet}$ are the input for the automatic description using regular expressions as in the methodology CI-IMS.

\section{Application of $I^{2} \mathrm{MC}$ to a nutritional intervention study}

\subsection{Nutritional intervention study}

The case study aims to analyze the Mediterranean diet effect on healthy people. In particular, it assesses if benefits associated with the traditional Mediterranean diet and virgin olive oil consumption could be mediated through changes in the expression of atherosclerosis-related genes as it was presented in [14,30].

First, inclusion criteria defined the participants recruited in the study. After, the selected participants were randomly split into the intervention groups (Mediterranean diet + Virgin olive oil, Mediterranean diet + Washed olive oil and Control). Data was collected before and after the diet intervention. Trial includes 89 healthy participants aged 20 to 50 years. They were considered healthy on the basis of a physical and biochemical examinations. Data comprised the biometrical characteristics, health and familiar history, background, socio-demographic data, current drug treatments, tobacco, alcohol and dietary habits, and physical activity recorded by a questionnaire. In

Table 5

Relation Table $(\mathscr{R})$ : behavior of all descriptors using the Sensitivity Analysis.

\begin{tabular}{|c|c|c|c|c|c|c|}
\hline & SuperClass & & & & & \\
\hline SubClass & $\bar{R}$ & $\bar{M}$ & $\bar{W}$ & $W$ & $M$ & $R / B$ \\
\hline $\bar{R}$ & Apparently Irrelevant & $\begin{array}{l}\text { Apparently Moderate } \\
\text { Irrelevant }\end{array}$ & $\begin{array}{l}\text { Apparently Weak } \\
\text { Irrelevant }\end{array}$ & $\begin{array}{l}\text { Apparently Weak } \\
\text { Inconsistency }\end{array}$ & $\begin{array}{l}\text { Apparently Moderate } \\
\text { Inconsistency }\end{array}$ & Apparently Inconsistency \\
\hline $\bar{M}$ & $\begin{array}{l}\text { Apparently Moderate } \\
\text { Irrelevant }\end{array}$ & $\begin{array}{l}\text { Apparently Weak } \\
\text { Irrelevant }\end{array}$ & $\begin{array}{l}\text { Apparently Limit } \\
\text { Irrelevant }\end{array}$ & $\begin{array}{l}\text { Apparently Limit } \\
\text { Inconsistency }\end{array}$ & $\begin{array}{l}\text { Apparently Weak } \\
\text { Inconsistency }\end{array}$ & $\begin{array}{l}\text { Apparently Moderate } \\
\text { Inconsistency }\end{array}$ \\
\hline $\bar{W}$ & $\begin{array}{l}\text { Apparently Weak } \\
\text { Irrelevant }\end{array}$ & $\begin{array}{l}\text { Apparently Limit } \\
\text { Irrelevant }\end{array}$ & Uncertain & Uncertain & $\begin{array}{l}\text { Apparently Limit } \\
\text { Inconsistency }\end{array}$ & $\begin{array}{l}\text { Apparently Weak } \\
\text { Inconsistency }\end{array}$ \\
\hline$W$ & Weak Specification & Limit Specification & Uncertain & Uncertain & Limit Inheritance & Weak Inheritance \\
\hline$M$ & Moderate Specification & Weak Specification & Limit Specification & Limit Inheritance & Weak Inheritance & Moderate Inheritance \\
\hline$R / B$ & Specification & Moderate Specification & Weak Specification & Weak Inheritance & Moderate Inheritance & Inheritance \\
\hline
\end{tabular}


Table 6

Action Table $(\mathscr{A})$ : actions to describe clusters.

\begin{tabular}{lllllll}
\hline \multicolumn{7}{c}{ SuperClass } \\
\hline SubClass & $\bar{R}$ & $\bar{M}$ & $\bar{W}$ & $W$ & $M$ & $R / B$ \\
\hline $\bar{R}$ & None & None & None & None & Inconsistency & Inconsistency \\
$\bar{M}$ & None & None & None & None & Inconsistency & Inconsistency \\
$\bar{W}$ & None & None & None & None & Both & Both \\
$W$ & None & None & None & None & Both & Both \\
$M$ & SubC & SubC & SubC & SubC & Both & Both \\
$R / B$ & SubC & SubC & SubC & SubC & Both & Both \\
\hline
\end{tabular}

addition, blood, urine and gene expression analysis were performed. The gene expression was performed in 47 subjects.

\subsection{Preprocessing}

In the data set, 242 variables were referred to the initial status of the participants. Preprocessing was conducted first, in collaboration with the experts. First of all variables' selection was done and variables providing useless information for clustering were cleaned (51 empty, 34 constant, 31 redundant, 25 irrelevant - i.e.: address). The 65 remaining variables were analyzed to identify null, incorrect or incoherent values and corrections were performed together with the experts (i.e. 43 variables referred to drug intakes and doses showed inconsistencies with variables regarding intake of drug families, as some participants declared consumption of an specific drug together with no-consumption of the corresponding drug family and vice versa). Once the inconsistencies of this part were treated, experts preferred to retain only the 7 variables referring the drug.

\subsection{Partitioning the individuals}

Different profiles of individuals depending on their characteristics at the beginning of the intervention are found.

The remaining variables basically referred to two semantic aspects. Thus, two thematic blocks were defined:

- The baseline characteristics block $(C)$ describes the health conditions. It contains information on biometrics, tobacco, socio-demographic, diseases, drugs and bio-markers.

- The habits block $(\mathrm{H})$ is related with life-style. It contains food habits and physical activity.

According to the proposed $\mathrm{I}^{2} \mathrm{MC}$, participants were clustered twice, once per block (Fig. 2), with Ward's method and the square of Gower's dissimilarity. The resulting clusters were crossed giving the final partition composed by 8 clusters. Since only 2 blocks are involved, a simple two way contingency table works. Resulting clusters gave the profiles of the participants regarding their baseline characteristics and their habits.

\subsection{Structural validation}

The same dataset was clustered under classical clustering methods for comparison:

- Hierarchical Clustering with Ward's method. The resulting dendrogram was cut in 6 and 12 clusters according to Calinsky-Harabasz Index.

- PAM: K was selected using a meta-algorithm that checked several values and proposed the one optimizing the Calinski-Harabasz Index. Here, the best cut in 8 clusters was retained.

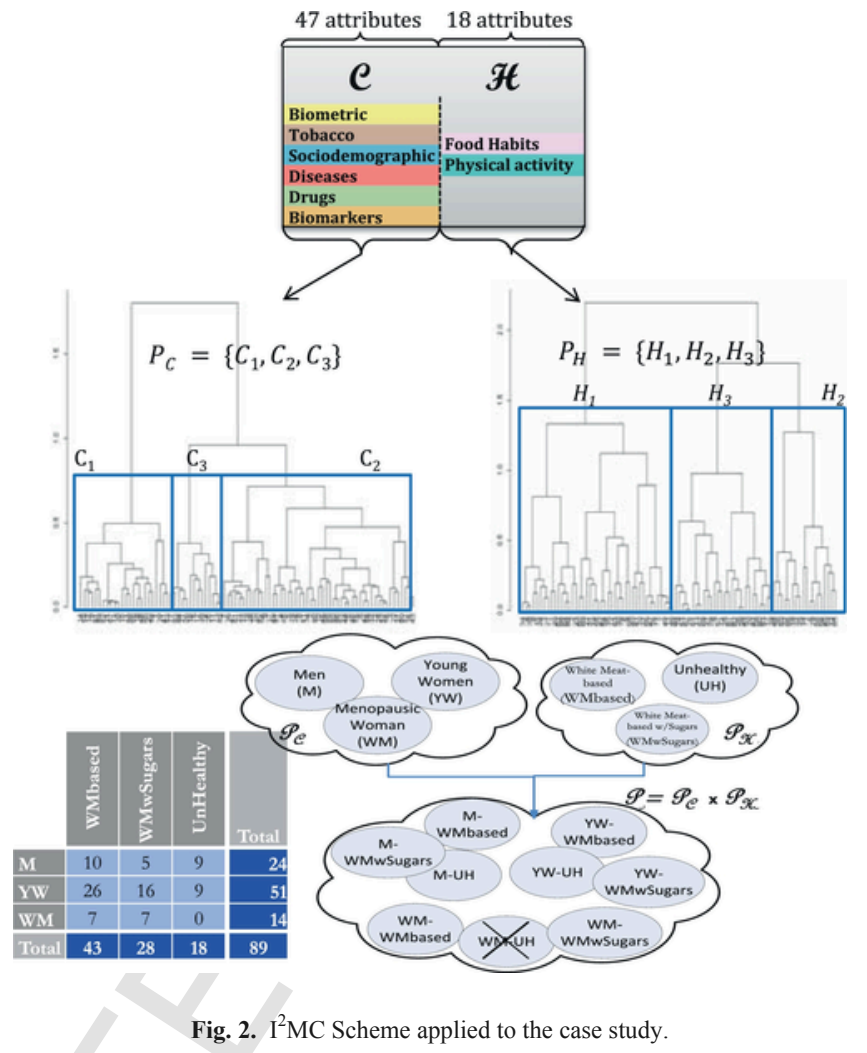

The IMC obtained the best results according to the Dunn-like index (Table 7). This indicates that IMC outperforms other methods from a structural point of view.

\subsection{Interpretability validation}

Additionally, interpretability of the clusters was also considered as it is critical from a decision-making point of view. Although the partitions seem structurally similar this do not necessarily imply that they are similar in terms of the participants conforming the clusters. In fact, as clustering methods explore the searching space under different heuristics, they get different local optima that might give different Cluster Interpretations.

The final description of the clusters was automatically generated with proposed methodologies of Section 3.9 for both IMC partition $\mathscr{P}$ and partition $H_{6}$, which is the second best in Table 7. Partition $\mathscr{P}$, being nested in the partitions of both thematic blocks used, was interpreted with the NCI-IMS with $\delta=0.25, \alpha=0.99, \varepsilon_{1}=0.2, \varepsilon_{2}=0.3$. Partition $\mathrm{HC}_{6}$ was interpreted through CI-IMS with the same parameters and with a minimum robustness of Weak-description $(r=W)$.

$\mathscr{P}$ shows clusters more easily interpretable from the clinical point of view. In particular, the final clusters describe 8 profiles composed

Table 7

Cluster Validity Comparison. As higher value of the Dunn-like index, better is the partition.

\begin{tabular}{lll}
\hline Method & $\mathrm{k}$ & Dunn-like \\
\hline Integrative MultiView Clustering $(P)$ & 8 & 0902 \\
Hierarchical Clustering $\left(H C_{6}\right)$ & 6 & 0896 \\
PAM & 8 & 0856 \\
Hierarchical Clustering $\left(H C_{12}\right)$ & 12 & 0809 \\
\hline
\end{tabular}


by three groups of participants (Man (M), Young Women (YW) and Older Women all in menopause (WM)) with three different diets: based in white meat, poor in fruits, red meat and legumes (WMbased); based in white meat with high consumption of fruits and commercial bakery (WMwSugars); and based in white meat, red meat and poor in vegetables (Unhealthy) (this is not followed by menopausal women). The labeling has been done according to the most descriptive and discriminative variables. For instance, the cluster Men (M) was named this way because the $95.8 \%$ of the individuals have the value man in variable gender (detailed profiles in Section 4.5.1).

From the point of view of interpretation, $\mathrm{HC}_{6}$ contains 6 clusters, with some confusing structure:

- Clusters $\left(G_{3}, G_{4}, G_{5}, G_{6}\right)$ are mainly composed by women, $G_{1}$ contains basically men. $G_{2}$ shows a significantly higher proportion of men than other clusters according to Test-Value, but this seems a contradiction with its $55 \%$ of men and $45 \%$ of women (Table 9). Some bio-markers and food are different in $G_{1}$ and $G_{2}$. However, the conditional distributions of some of the variables strongly overlap (F2 $\alpha$-Isoprostanes, C-Reactive Protein, HDL, triglycerides, Tissue Plasminogen Activator) and do not support a neat characterization towards distinguishable profiles.
- Women clusters seem to be also confusing. $G_{5}$ and $G_{6}$ seem to contain significantly older persons than $G_{3}$ and $G_{4}$. However, $G_{5}$ ranges from 20 to 70 years (see Table 9). The degree of overlapping of the conditional distributions is really high to assume real differences in profiles, in spite of significantly $p$-values obtained (glucose, Interleukin 10, F2 $\alpha$-Isoprostanes).

- Dietary patterns show high variability in some foods that are no significant in clusters.

The CPGs of Tables 8 and 9 show these confusing profiles for the $H C_{6}$ partition in comparison with partition $\mathscr{P}$, which shows a clearer definition of values among clusters.

\subsubsection{Profiles of $(\mathscr{P})$ derived from $I^{2} M C$}

M-WMbased: men group, taller and heavier (weight, waist). Their blood pressure is higher (systolic, diastolic) and lower heart rate. Most are married. No menopause cases and they consider themselves less stressful. No incidence of other diseases. Low consumption of drugs. Lower level of HDL, Interleukin 6 and Interleukin 10. Higher levels of F2 $\alpha$-Isoprostanes, Monocyte Chemotactic Protein-1, Tyrosol, OHTyrosol, MOH Tyrosol. With a diet white meat based. They consume more butter than general sample and less gas drinks and commercial bakery. They practice more exercise, specially intense, but they do less household chores.

Table 8

Class Panel Graphs for partition $\mathscr{P}$ (IMC).

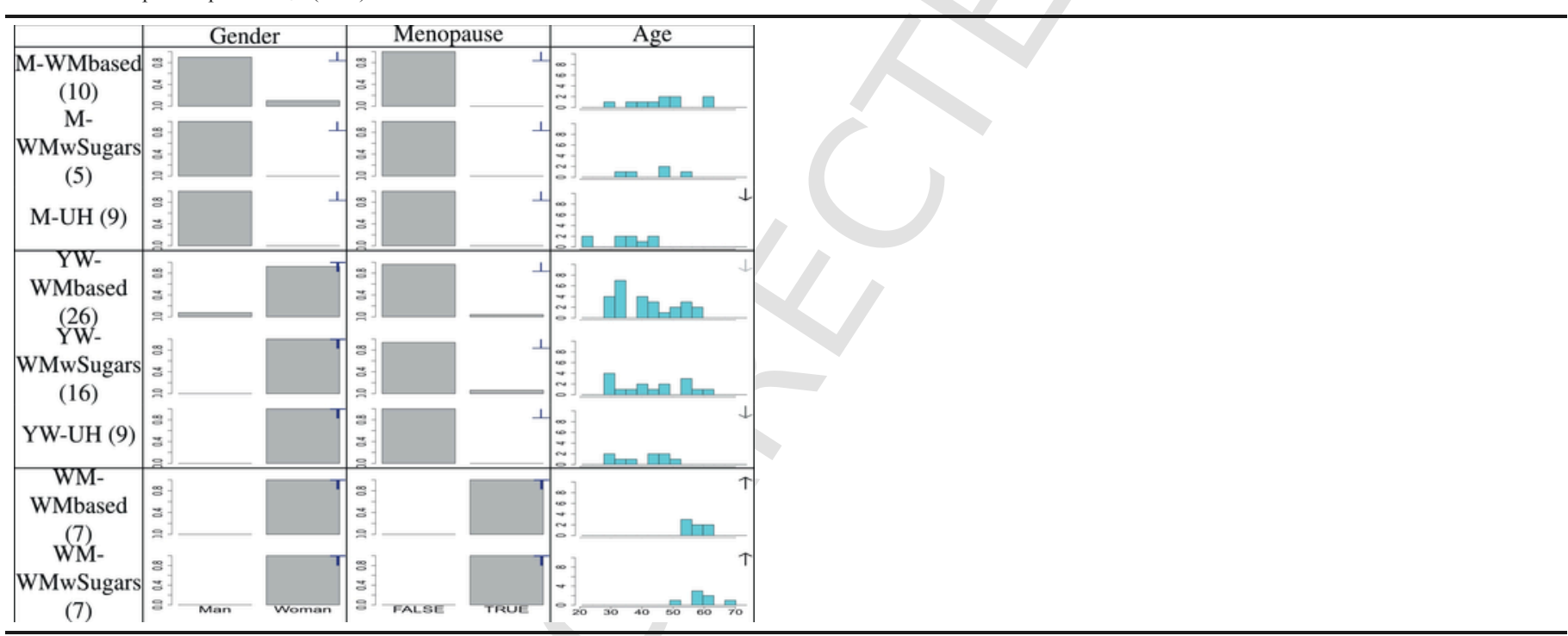

Table 9

Class Panel Graphs for partition $\mathrm{HC}_{6}$.

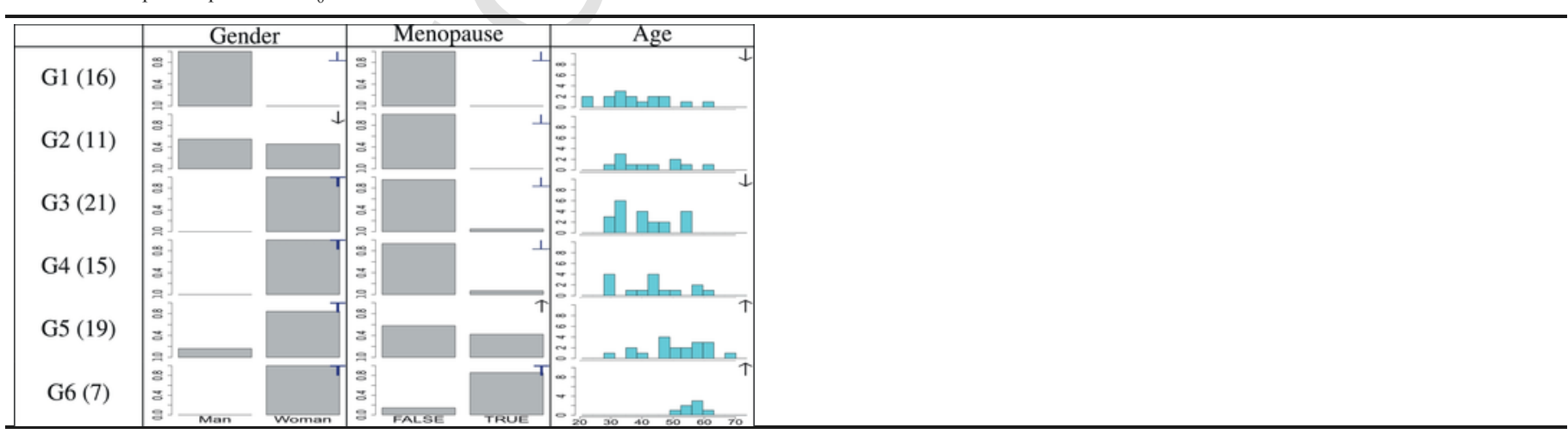


M-WMwSugars: men group that are older, taller and heavier (weight, BMI, waist). With higher systolic and diastolic pressure. Most have never smoked. Most are married. They consider themselves less stressful. Higher incidence of bone fracture and dyspnea. Low drugs consumption but higher intake of "other" drugs. Lower levels of LDL, HDL, sCD40 Ligand, C-Reactive Protein and Tyrosol. Higher levels of glucose, triglycerides, Monocyte Chemotactic Protein-1, sP-selectin and Tissue Plasmingen Activator. With a white meat based with sugars diet. They consume more legumes and less red meat and they drink more wine and less gas drinks. They practice more exercise but less intense exercise and less at home.

M-UH: Younger men group that are taller and heavier (weight, BMI, waist). With higher systolic pressure and lower heart rate. Lower proportion of non-smokers. Higher proportion of singles. No menopause cases. They consider themselves less stressful. No incidence of other diseases. Lower levels of cholesterol, LDL, HDL and 8-Oxoguanine. Higher levels of triglycerides, F2 $\alpha$-Isoprostanes, sP-selectin, sCD40 Ligand and Tissue Plasminogen Activator. With the Unhealthy diet. They consume more red meat and gas drink and less butter than the general sample. They practice less exercise in general.

WY-WMbased: group composed mainly by women. They are younger and thinner (weight, BMI, waist). Their systolic and diastolic pressure is lower. Higher proportion of smokers than other groups. No menopause in general. They consider themselves more stressful. No incidence of other diseases. They take more painkillers. Lower levels of glucose, triglycerides, Monocyte Chemotactic Protein-1 and higher of HDL and C-Reactive Protein. With a white meat based diet, they eat more vegetables and fish. They consume little butter and wine.

WY-WMwSugars: woman group which are smaller and thinner (weight, BMI, waist). Lower systolic pressure. Contains the unique depressed person and no incidence of other diseases. Lower levels of cholesterol, LDL, triglycerides, Oxidized LDL, C-Reactive Protein and Tissue Plasminogen Activator. Higher levels of Interferon- $\gamma$, Tumor necrosis factor- $\alpha$, OHTyrosol and MOHTyrosol. With a diet white meat with sugars. They consume more nuts and less red meat and legumes. They practice more intense and total exercise.

WY-UH: younger women group that are smaller and with lower systolic and diastolic pressure. No menopause cases. They are more stressful. No incidences of other diseases. Low consumption of drugs but slightly higher of "other" drugs. Lower levels of glucose, cholesterol, LDL, triglycerides, Oxidized LDL, Tissue Plasminogen Activator and Interleukin 10. Higher levels of F $2 \alpha$-Isoprostanes, 8-Oxoguanine, Tumor necrosis factor- $\alpha$, Soluble cell adhesion molecules-1, Interleukin 6 and Tyrosol. With an Unhealthy diet and they consume less olive oil, gas drinks and legumes and use more butter. They do more homework and less exercise in general.

WM-WMbased: older women which are shorter and thinner (weight). Higher diastolic pressure and heart rate. Most are married. All have menopause. They consider themselves more stressful. They have higher incidence of cancer with no other incidences of diseases. They take more "other" drugs. Higher levels of glucose, cholesterol, LDL, HDL, Oxidized LDL, C-Reactive Protein, Interleukin 8 and Interleukin 10. Lower levels of F2 $\alpha$ Isoprostanes, sP-selectin sCD40 Ligand and Tyrosol. With a white meat based diet and rich in vegetables and poor in butter, wine, commercial bakery and nuts. They do more light exercise but do less total exercise in general.

WM-WMwSugars: older women group which are shorter and heavier (BMI, waist). They have higher blood pressure and heart rate. Lower proportion of smokers and singles. All have menopause. No incidence of other diseases. They take more painkillers (NSAID). They have higher levels of glucose, cholesterol, LDL, triglycerides,
Oxidized LDL and Interleukin 10. Lower levels of HDL, Monocyte Chemotactic Protein-1, sP-selectin, sCD40 Ligand, C-Reactive Protein and Tyrosol. With a white meat base with sugars diet. They consume less vegetables, red meat, gas drinks and wine. They eat more nuts. They do less intense exercise and more homework.

\section{Conclusions and future work}

This research has been tested within the scope of pre-post nutritional studies. This work has been motivated by the inherent difficulties to use classical approaches on this type of studies in current data sets, more and more complex, as referred in Section 1.

The goal is to find a set of profiles describing the types of participants of the trial assay as a reference to relate, in a second step of the research, the adherence to and the effects of the intervention in each type of individual. For that reason, getting a meaningful set of profiles was a main target of this work.

For this goal, the Interpreted Integrative Multiview Clustering $\left(\mathrm{I}^{2} \mathrm{MC}\right)$ has been introduced. With a main step of IMC followed by interpretation of the resulting nested partitions.

On the one hand, $\mathrm{I}^{2} \mathrm{MC}$ reduces the computational cost of analyzing high dimensional data; on the other hand, it provides more interpretable clusters, and produces automatic conceptualization of clusters. Data is decomposed into meaningful subsets using domain knowledge to define the views. Individuals are repeatedly clustered per each thematic blocks. Multiview principle is used to simplify the problem structure and to enable local complex models. A further cross-clustering step integrates knowledge from the multiview in a single typology, by catching relationships between meaningful concepts. The $\mathrm{I}^{2} \mathrm{MC}$ methodology is general and permits as many views as desired (which is not considered in other proposals such as [6]). As all methods oriented to interpretation, $\mathrm{I}^{2} \mathrm{MC}$ assumes an active participation of the experts all along the process in different steps: determining the data set to be analyzed, determining reasonable values for the input parameters according to the costs of wrong decisions, in the preprocessing decisions, building the thematic blocks according to the semantics of the variables, conceptualizing the resulting clusters on the basis of the automatic interpretations provided by the $\mathrm{I}^{2} \mathrm{MC}$ method, validating the cluster's usefulness and giving meaningful names to each cluster (for future use). The complete methodology relies on 4 parameters $\delta, \alpha, \varepsilon_{1}, \varepsilon_{2}$ all of them related with the treatment of uncertainty assumed by the method, and it is recommendable to keep the two former small and the two later high. However, the threshold of uncertainty in results that can be assumed by the experts is a key point to evaluate how bigger or smaller this parameters must be. The smaller is $\delta$, the few basic descriptors will be identified in the clusters; the smaller is $\alpha$, the few ordinary cluster descriptors will be identified in the clusters; the smaller are $\varepsilon_{1}, \varepsilon_{2}$ the sensitivity analysis of the ordinary cluster descriptors will happen in a closer neighborhood around the current sample size, so, the less guarantee of robustness for cluster descriptors is given.

The method works well with small $\beta$. When the $\beta$ is too high, it means that many separate views are identified in the data set. The final partition, which is the result of a Cartesian product, can be mapped into a $\beta$-dimensional cross-table that increases sparsity as $\beta$ increases. In this case, some thematic packs can be merged together in a single view to reduce $\beta$. The proposed method must be unable to identify cluster descriptors when the variability inside the clusters is too high and the sample size too small. Then, the statistical tests will result non-significant and no descriptors will be identified for the clusters. However, this is rather improbable, since high overlapping between clusters and high variability inside the clusters corresponds 
better to the situation in which clusters are artificial and no real clusters exists, and this will produce non-significant Calinski-Harabasz results to cut the dendrograms. Thus, provided that the Multiview Clustering is performed on the basis of a Hierarchical Clustering methods with Calinski-Harabasz as the criterion to decide the number of clusters, it is difficult to meet this scenario.

$\mathrm{I}^{2} \mathrm{MC}$ includes a novel Cluster Interpretation process NCI-IMS which uses the new Cluster Interpretation methodology CI-IMS. NCI-IMS is suitable for understanding the profiles of real sample data and to deal with the consistency of the interpretation of nested partitions, both inter clusters and inter partitions.

NCI-IMS is required because the resulting partition of IMC is nested in the partitions obtained by the several views considered. NCI-IMS handles the possible inconsistencies between the profile of a nested class (subclass) and the profile of its corresponding superclass (see Section 3.11). NCI-IMS uses the resulting profiles of the proposed CI-IMS (Section 3.9) by taking advantage of the description-power that graduates its robustness. Given a partition and a set of variables, CI-IMS obtains the description of each cluster according to the robustness of variables' significance. This methodology introduces the Sensitivity Analysis which determines the description-power that graduates the strength of the descriptor. This Sensitivity Analysis uses the proposed generalized Test-Value that can be assessed with variable cluster sizes. Also, the basic descriptors are introduced for quasi-constant variables that play an important role in the description of the clusters, but cannot be assessed by statistical tests using variances in the denominator. A formalization of the concept of cluster descriptor is introduced in this work as the reference language to evaluate over clusters and the resulting relevant cluster descriptors obtained with the Sensitivity Analysis can be translated to natural language conceptualizations based on regular expressions.

$\mathrm{I}^{2} \mathrm{MC}$ has been applied over the data of a real nutritional intervention study. State of individuals at the beginning of the study have been classified under two views (baseline characteristics and habits). Eight different patterns were obtained $(\mathscr{P})$ and compared with those provided by Ward's $\left(H_{6}\right)$ or PAM method. From a structural point of view, $\mathscr{P}$ is the best according to the Dunn-like index. Also, according to experts, the interpretation given by $\mathrm{I}^{2} \mathrm{MC}$ seems clearer than the interpretation of $\mathrm{HC}_{6}$. The fact that $\mathrm{HC}_{6}$ clusters are build with all variables at once leads to clusters that seem to mix some concepts. Comparing each cluster of $H C_{6}$ with the IMC clusters, $G_{6}$ is practically equivalent to WM-WMbased and $G_{1}$ to M-UH. $G_{5}$ mixes women with or without menopause that follow a WMwSugars diet. $G_{3}$ contains young women mainly with WMbased or UH diet and most of the YW-UH are in this clusters.

The original dataset contained 256 variables. $\mathrm{I}^{2} \mathrm{MC}$ provided an automatic interpretation of the 8 discovered profiles expressed in no more than 30 variables per cluster, which are significant for the cluster. A short observation of these descriptions leads the expert to a clear comprehension of which are the relevant differences among the discovered profiles.

From a methodological point of view a benchmark is being performed to assess theoretical properties of our proposal by comparing results on different data sets with different structures, as well as a synthetic data set with well known structure to be used as a ground-truth.

Also, authors are working on objective metrics that can assess the quality of an interpretation. Currently, the lack of this kind of indicators reduces evaluation of interpretations to the opinions of experts when they try to understand them. This is a valuable criteria, as it is pointing to further usability of the discovered profiles, but further findings in this topic will help.
Currently, $\mathrm{I}^{2} \mathrm{MC}$ is being used to profile participants at the end of the intervention, and modeling the intervention adherence and effect over each type of individual is in progress. As gene expression is available, relationships among intervention effect, type of individuals obtained in this work and genetic conditions can be studied. Thus, further knowledge on the role of genes in metabolizing nutrients can be obtained.

This is an interesting research field since more and more resources are devoted to extract more knowledge for human genome, and there is an increasing activity in the area. The presented strategy opens the door for personalized analysis of how diet can benefit on individuals in the future.

\section{Acknowledgment}

This work has been partially supported by the project Diet4You (TIN2014-60557-R) funded by Spanish Government.

\section{References}

[1] A. Abdullin, O. Nasraoui, Clustering heterogeneous data sets, Web Congress (LA-WEB), 2012 Eighth Latin American, IEEE, 20121-8.

[2] L. Afman, M. Müller, Nutrigenomics: from molecular nutrition to prevention of disease, J. Am. Diet Assoc. 106 (4) (2006) 569-576.

[3] K. Barnard, P. Duygulu, D. Forsyth, Clustering art, Proc. of CVPR (2001), 2, 2001. II-434-II-441

[4] P. Berkhin, A survey of clustering data mining techniques, Grouping Multidimensional Data, Springer, 200625-71.

[5] J.C. Bezdek, N.R. Pal, Some new indexes of cluster validity, Syst. Man Cybern. Part B 28 (3) (1998) 301-315.

[6] S. Bickel, T. Scheffer, Multi-view clustering, ICDM, 4, 200419-26.

[7] T. Caliński, J. Harabasz, A dendrite method for cluster analysis, Commun. Stat.-Theory Methods 3 (1) (1974) 1-27.

[8] W. Cecere, D.A. Abreu, A method for improving list building: cluster profiling, Proceedings of the Survey Research Methods Section, ASA, 2010.

[9] B. Clarke, E. Fokoue, H.H. Zhang, Principles and Theory for Data Mining and Machine Learning, Springer Science \& Business Media, 2009.

[10] P. Cortez, M.J. Embrechts, Using sensitivity analysis and visualization techniques to open black box data mining models, Inf. Sci. 225 (0) (2012) 1-17.

[11] M. D'Orazio, StatMatch: Statistical Matching, 2012. R package version 1.0.5

[12] R.O. Duda, P.E. Hart, D.G. Stork, Pattern Classification, Wiley, 2012.

[13] J.C. Dunn, Well separated clusters and optimal fuzzy partitions, J.Cybern. 4 (1) (1974) 95-104.

[14] R. Estruch, E. Ros, et al., Primary prevention of cardiovascular disease with a mediterranean diet, NEJM 368 (14) (2013) 1279-1290.

[15] K. Gibert, Automatic generation of classes interpretation as a bridge between clustering and decision making, Int. J. Multicriteria Decis. Making 4 (2) (2014) $154-182$.

[16] K. Gibert, D. Conti, D. Vrecko, Assisting the end-user in the interpretation of profiles for decision support. An application to wastewater treatment plants, Environ. Eng. Manage. J. 11 (5) (2012) 931-944.

[17] K. Gibert, A. García-Rudolph, et al., The role of KDD support-Interpretation tools in the conceptualization of medical profiles: an application to neurorehabilitation, Acta Informatica Medica 16 (4) (2008) 178-182.

[18] K. Gibert, R. Nonell, J. Velarde, M.M. Colillas, Knowledge discovery with clustering: impact of metrics and reporting phase by using klass, Neural Netw. World 4 (2005) 319-326.

[19] K. Gibert, G. Rodríguez-Silva, R. Annicchiarico, Post-processing: bridging the gap between modelling and effective decision-support. the profile assessment grid in human behaviour, Math. Comput. Model. 57 (7-8) (2013) 1633-1639.

[20] K. Gibert, M. Sànchez-Marrè, J. Izquierdo, A survey on pre-processing techniques in the context of environmental data mining, Artif. Intell. Commun. 40 (2016) 559.

[21] K. Gibert, B. Sevilla-Villanueva, M. Sànchez-Marrè, The role of significance tests in consistent interpretation of nested partitions, J. Comput. Appl. Math. 292 (2016) 623.

[22] K. Gibert, A. Valls, M. Batet, Introducing semantic variables in mixed distance measures: impact on hierarchical clustering, Knowl. Inf. Syst. 40 (2014) 559

[23] K.C. Gowda, E. Diday, Symbolic clustering using a new similarity measure, Syst. Man Cybern. IEEE Trans. 22 (2) (1992) 368-378.

[24] J. Gower, A general coefficient if similarity and some of its properties., Biometrics 27 (1971) 857-874.

[25] M. Halkidi, Y. Batistakis, M. Vazirgiannis, On clustering validation techniques, J. Intell. Inf. Syst. 17 (2) (2001) 107-145. 
[26] C. Hennig, fpc: Flexible Procedures for Clustering, 2013. R package v. 2.1-5

[27] K. Hulshof, M. Wedel, et al., Clustering of dietary variables and other lifestyle factors (dutch nutritional surveillance system), JECH 46 (4) (1992) 417-424.

[28] M. Ichino, H. Yaguchi, Generalized Minkowski metrics for mixed feature-type data analysis, IEEE Tr SMC 22 (2) (1994) 146-153.

[29] L. Kaufman, P. Rousseeuw, Clustering by means of medoids, Stat. Data Anal. L1-Norm Relat. Methods (1987) 405-416.

[30] V. Konstantinidou, M.I. Covas, et al., In vivo nutrigenomic effects of virgin olive oil polyphenols within the frame of the mediterranean diet: a randomized controlled trial., FASEB J. 24 (7) (2010) 2546-2557.

[31] L. Lebart, M. Piron, A. Morineau, Statistique Exploratoire Multidimensionnelle, 3, Dunob, 2000.

[32] D. Li, P. Shafto, Bayesian hierarchical cross-clustering, AISTATS, 15, 2011443-451.

[33] N.R. Pal, J. Biswas, Cluster validation using graph theoretic concepts, Pattern Recognit. 30 (6) (1997) 847-857.

[34] H. Ralambondrainy, A Conceptual Version of the K-means Algorithm, Lifetime Learning Publications, Belmont, California, 1995

[35] M. Sànchez-Marrè, I.R. Roda, Q. Comas, L'eixample distance: a new similarity measure for case retrieval, CCIA'98, FAIA 14-15 (1998) 246-253.
[36] M. Sarstedt, E. Mooi, A Concise Guide to Market Research: The Process, Data, and Methods using IBM SPSS Statistics, Springer Verlag, 2011.

[37] B. Sevilla-Villanueva, A Methodology for Pre-Post Intervention Studies: An Application for a Nutritional Case Study, Universitat Politècnica de Catalunya BarcelonaTECH, 2016, Ph.d. programme in artificial intelligence.

[38] B. Sevilla-Villanueva, K. Gibert, M. Sànchez-Marrè, Post-processing the class panel graphs: towards understandable patterns from data., Frontiers in Artificial Intelligence and Applications, v. 256, 2013215-224.

[39] B. Sevilla-Villanueva, K. Gibert, M. Sànchez-Marrè, Identifying nutritional patterns through integrative multiview clustering, Frontiers in Artificial Intelligence and Applications, v. 277, 2015185-194.

[40] M. Siponen, J. Vesanto, O. Simula, P. Vasara, An approach to automated interpretation of SOM, Advances in Self-Organising Maps, Springer, 200189-94.

[41] S. Swaminathan, T. Thomas, S. Yusuf, M. Vaz, Clustering of diet, physical activity and overweight in parents and offspring in South India, Eur. J. Clin. Nutr. 67 (2) (2013) 128-134.

[42] J.H. Ward Jr, Hierarchical grouping to optimize an objective function, J. Am.Stat.Assoc. 58 (301) (1963) 236-244.

[43] X. Yin, J. Han, P. Yu, Cross-relational clustering with user's guidance, ACM SIGKDD, 2005344-353. 